\title{
Object appearance, disappearance, and attention prioritization in real-world scenes
}

\author{
JAMES R. BROCKMOLE and JOHN M. HENDERSON \\ Michigan State University, East Lansing, Michigan
}

\begin{abstract}
We examined the prioritization of abruptly appearing and disappearing objects in real-world scenes. These scene changes occurred either during a fixation (transient appearance/disappearance) or during a saccade (nontransient appearance/disappearance). Prioritization was measured by the eyes' propensity to be directed to the region of the scene change. Object additions and deletions were fixated at rates greater than chance, suggesting that both types of scene change are cues used by the visual system to guide attention during scene exploration, although appearances were fixated twice as often as disappearances, indicating that new objects are more salient than deleted objects. New and deleted objects were prioritized sooner and more frequently if they occurred during a fixation, as compared with during a saccade, indicating an important role of the transient signal that often accompanies sudden changes in scenes. New objects were prioritized regardless of whether they appeared during a fixation or a saccade, whereas prioritization of a deleted object occurred only if (1) a transient signal was present or (2) the removal of the object revealed previously occluded objects.
\end{abstract}

The goal-directed orientation of attention to known targets in a visual display is sometimes disrupted by the presence of distinctive features or objects that attract attention, even when such features are irrelevant to the observer's goals, an effect called attention capture. Depending on the goals of the observer (see Bacon \& Egeth, 1994; Folk, Remington, \& Johnston, 1992), several kinds of stimuli, including the abrupt appearance of a new object (see, e.g., Yantis \& Jonides, 1984), the sudden disappearance of an existing object (e.g., Theeuwes, 1991), and objects characterized by unique color, shape (Theeuwes, 1994), or movement (e.g., Franconeri \& Simons, 2003) have been shown to capture attention. Generally, previous demonstrations of attention capture have assessed the ability of a single unique but irrelevant item to disrupt visual search in simple arrays of letters or shapes.

Given that the goal of attention capture studies is to describe a general principle of attention allocation to visual objects, it is vital that such effects be observed with more naturalistic stimuli. Real-world scenes possess a degree of visual and semantic complexity that is absent in simple stimulus arrays. Scenes are collections of heterogeneous visual features, and therefore, no object serves as the single

This research was supported by the National Science Foundation (BCS-0094433 and IGERT Grant ECS-9874541), the Army Research Office (W911NF-04-1-0078), and a Strategic Partnership Grant from the MSU Foundation. We thank Steve Franconeri, Derrick Watson, and an anonymous reviewer for their helpful comments on an earlier version of the manuscript. We also thank Brendon Hsieh for taking the photographs used in this study, Elyzabeth Marshall for her help with data collection, and Michael Mack for his technical assistance. Address correspondence concerning this article to J. R. Brockmole, Department of Psychology, University of Edinburgh, 7 George Square, Room G.30, Edinburgh EH8 9JZ, Scotland (e-mail: james.brockmole@ed.ac.uk). unique item. In addition, even objects that do not constitute a specific search target may provide useful information about the target's location. Thus, no particular object or feature is known, a priori, to be irrelevant to the observer's task. Real-world scenes, therefore, afford viewing conditions quite dissimilar to those provided by the kinds of circumstances that have thus far been shown to produce capture effects. On the basis of prior research, then, it is unclear whether the same mechanisms that drive attention to new or unique objects in simple stimulus arrays also operate under more naturalistic viewing conditions.

In a recent report, Brockmole and Henderson (2005) undertook the first examination of attention capture in realworld scenes by investigating the impact of an abruptly appearing new object on attention allocation, as assessed by gaze behavior. Capture was measured by the eyes' propensity to be directed to the new object once it had appeared (Theeuwes, Kramer, Hahn, \& Irwin, 1998). In an effort to consider the influence of new objects on gaze divorced from the low-level transient signals that often accompany the appearance of an object, objects appeared either during a fixation, so that they retained their transient status, or during a saccade, which because of saccadic suppression, eliminated the transient signal. In both conditions, the eyes were directed to the new object at rates greater than would be expected by chance. However, new objects that appeared during fixations were fixated in half the time and twice as often as those that appeared during saccades, indicating a prioritizing effect specific to the transient signal. In addition, prioritization of new objects during saccades, but not during fixations, was affected by manipulations of memory for the scene. These results suggested that new objects appearing in real-world scenes receive attentional prioritization via two mechanisms: Objects with a transient signal capture attention 
immediately and with high frequency, whereas without a transient signal, new objects are prioritized over several saccades as memory processes detect the change.

In the present study, we investigated the degree to which the disappearance of an object produces capture, as compared with that generated by the appearance of an object. Relative to object appearance, the evidence for attention capture by the removal of an object is more equivocal, and no previous research has been done to examine whether the disappearance of an object from a visual display affects the top-down guidance of gaze. Although some research suggests that transient offsets attract attention with the same efficacy as do transient onsets (Miller, 1989; Theeuwes, 1991; Watson \& Humphreys, 1995), other studies suggest that transient offsets are less adept at capturing attention than are onsets. For example, when both onsets and offsets are simultaneously present in a visual display, the onset reveals the predominant capture effect, suggesting that onsets enjoy priority over offsets (Jonides \& Yantis, 1988; Yantis \& Jonides, 1984). In addition, the time course of capture elicited by onsets and offsets appears to be different. By varying the stimulus onset asynchrony between an onset or offset and the revelation of a search target, researchers have determined that attention is disengaged from an offset more quickly than from an onset (Chastain \& Cheal, 1999; Pratt \& Hirshhorn, 2003). Together, the latter results suggest that offsets in a visual display are less salient than onsets.

Given the potential differences between onsets and offsets, the present experiment had two goals. First, we aimed to compare any prioritization for object appearance versus disappearance in natural scenes. Observers viewed photographs of scenes as their eye positions were monitored. The location of eye fixation was taken as an index of the locus of attention within the scene. In separate conditions, a single object was either added to or deleted from each scene during viewing (see Figure 1). Prioritization is revealed if the region containing the appearance or disappearance becomes the most salient region in the scene. We operationalized the speed of prioritization as the number of saccades executed between the occurrence of the object's appearance or disappearance and an observer's first fixation on the changed scene region. We operationalized the frequency of detection as the overall probability that the scene region containing the change was fixated after its occurrence. We will reserve the term capture to refer to fast and immediate prioritization.

Second, like Brockmole and Henderson (2005), we investigated the importance of a transient signal in guiding the orientation of attention to an object's appearance and disappearance. Objects appeared and disappeared either during a fixation or during a saccade. The fixation condition enabled us to contrast the ability of these changes to reflexively capture attention and to determine whether they are equal in their salience. The saccade condition allowed us to examine whether memory supports the prioritization of nontransient object deletions from a scene, as well as nontransient additions. According to Brockmole and Henderson, memory guides attention and the eyes to a nontransient object's appearance by supporting a com-

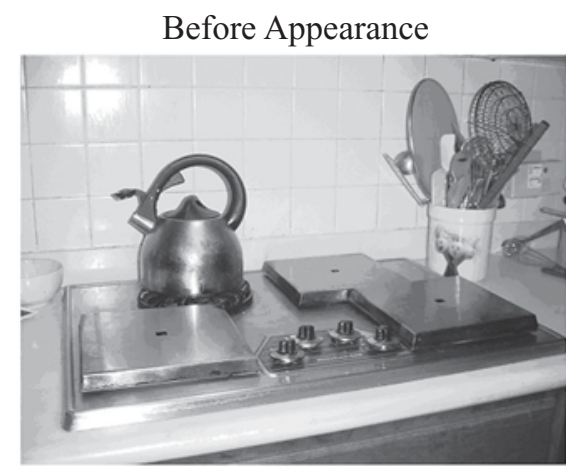

Before Disappearance

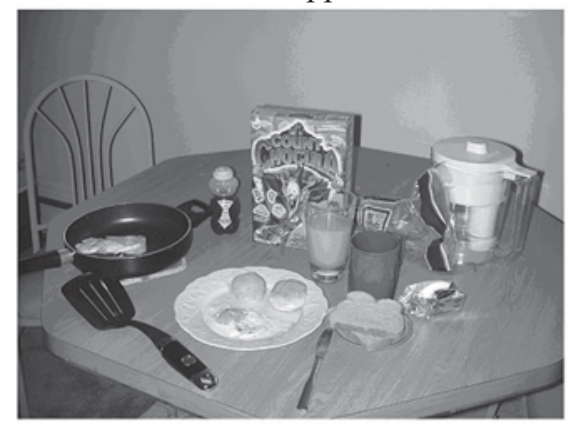

After Appearance

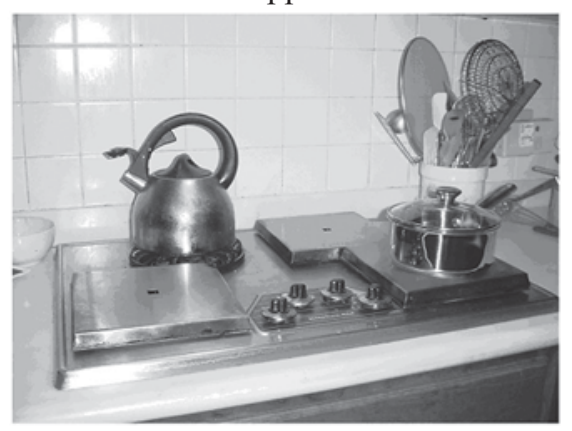

After Disappearance

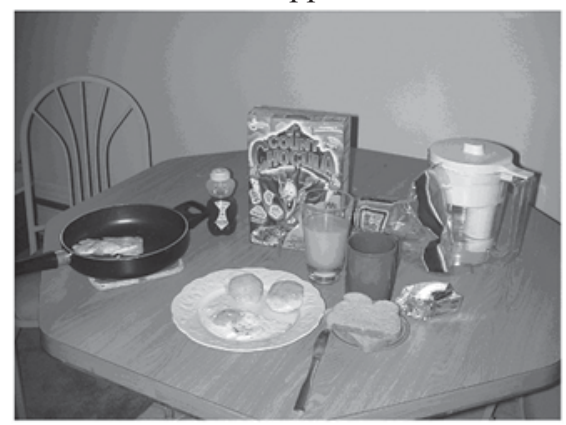

Figure 1. Example scenes used in this study depicted both before and after object appearance (top panel) or disappearance (bottom panel). In these examples, the pot and the spatula are the critical objects. 
parison of the current view of a scene with a prior view. When a difference is noted, the new object is localized. With such a process, in principle, the disappearance of an object ought to be detected as well. However, the perceptto-memory comparison may be more likely to detect the addition of an object, as compared with the deletion of one. In previous tests of memory, changes to a visual scene that incorporated the addition of an element in a memory paradigm were detected better than changes that involved the deletion of an element (Hearst, 1991; Henderson, Williams, Castelhano, \& Falk, 2003; Pezdek et al., 1988). Comparing the prioritization of new and deleted objects in the saccade condition can, therefore, allow us to determine how memory for a scene can be used to locate different types of scene changes.

\section{METHOD}

Sixteen Michigan State University undergraduates were shown 30 full-color photographs depicting real-world scenes. Two photographs of each scene were taken that differed only in the presence or absence of a single object (see Figure 1 for examples). ${ }^{1}$ The photographs were digitally edited to eliminate minor differences in shadow and spatial displacement. The photographs were displayed at a refresh rate of $100 \mathrm{~Hz}$ on a CRT at a resolution of $800 \times 600$ pixels in 24-bit color and subtended $37^{\circ}$ horizontally and $27.5^{\circ}$ vertically at a viewing distance of $57 \mathrm{~cm}$. An ISCAN ETL-400 pupil and corneal reflection tracking system, accurate to within $0.5^{\circ}$ of visual angle, sampled eye position at $240 \mathrm{~Hz}$. Chin and forehead rests were used to stabilize viewing position and distance.

The participants were divided into two scene change conditions. In the new-object condition, an object was added to the scene during viewing, replicating Brockmole and Henderson (2005). In the deleted-object condition, an existing object was removed from the scene. The same object in each scene served as the appearing or the disappearing object. All the participants were instructed to memorize each scene in preparation for a subsequent test in which they would discriminate the studied scenes from scenes in which a detail of a single object would be altered (in actuality, this test was never given). No explicit instructions concerning the appearance or disappearance of new objects were given. ${ }^{2}$

The participants began the experimental session by completing a calibration routine that mapped the output of the eyetracker onto display position. Calibration was constantly monitored throughout the experiment and was adjusted when necessary. The participants began each trial by fixating a dot in the center of the display. When they were ready to view the stimulus, a photograph was displayed for $10 \mathrm{sec}$. Depending on the participant's condition assignment, a single object was either deleted from or added to the scene by changing the presented photograph to its associated counterpart. Phenomenologically, this change looked like the simple addition or deletion of an object in the scene.

Scene changes were introduced the first time the eyes crossed the midline of the display after $5 \mathrm{sec}$ had elapsed since the beginning of the trial, as in Brockmole and Henderson (2005). ${ }^{3}$ When the change was to appear during a saccade, its occurrence was coincident with the eyes crossing the midline following the 5-sec interval. In this condition, the eyes were still moving when the change occurred. When the change was to appear during a fixation, it occurred $100 \mathrm{msec}$ after the eyes crossed the midline following the 5-sec interval. This $100-\mathrm{msec}$ delay was long enough to allow the critical saccade to terminate, but short enough that a subsequent saccade could not be launched. In this condition, the addition or deletion occurred when the eyes were still. ${ }^{4}$

\section{RESULTS AND DISCUSSION}

For each scene, a region of interest was defined by an imaginary bounding box surrounding the critical object that appeared or disappeared. Fixations were sorted according to whether or not they fell within these regions. Analyses focused on the four fixations immediately following the scene change (denoted Fixations 1-4, respectively). We refer to this as the ordinal fixation position. Fixation 1 denotes the termination of the first saccade launched after the critical change occurred to the scene and, therefore, represents the first fixation that could be influenced by the change. Analyses focused on how often and how quickly the critical region was fixated. If new or deleted objects capture attention, the eyes should be directed to the location of the scene change with greaterthan-chance probability and very quickly after the change takes place. If new or deleted objects are prioritized without capture, the eyes should be directed to the location of the change with greater-than-chance probability, but not as certainly and more slowly.

\section{Appearing Objects}

Number of eye movements to first fixation on new objects. On average, new objects appearing during a fixation were viewed 1.57 saccades after their appearance. Those that appeared during a saccade were viewed 3.01 saccades after the onset. The difference between these conditions was reliable $[t(7)=5.65, p<.001]$. Figure $2 \mathrm{~A}$ illustrates the probability that the new item was first fixated at each of the ordinal fixation positions, given that it was fixated at all, according to whether it appeared during a fixation or a saccade. The probability that the first look to the new object occurred at each of the four ordinal fixation positions differed $\left[F(3,21)=25.7, M S_{\mathrm{e}}=2.02, p<\right.$ $.001]$, and these differences were not equal in the saccade and the fixation conditions $\left[F(3,21)=38.4, M S_{\mathrm{e}}=1.60\right.$, $p<.001] .{ }^{5}$ In the fixation condition, $74 \%$ of first looks to the new object occurred at Fixation 1, an observer's first opportunity to do so. This was followed by a rapid decline in the probability of a first look at each of the next ordinal fixation positions. Only $6 \%$ of first looks to the onset occurred at Fixations 3 and 4, combined. In contrast, in the saccade condition, $16 \%$ of first looks to the new object occurred at Fixation 1. The greatest percentage of first looks, 39\%, occurred at Fixation 2. Almost half of first looks (45\%) still occurred at Fixations 3 and 4, combined. In terms of cumulative probabilities, as many first looks to the new object occurred over the course of three fixations in the saccade condition as in the very first fixation in the fixation condition. In essence, it took longer to detect the new object in the saccade condition.

Probability of fixating new objects. How frequently was the appearance of a new object detected? Figure 2B illustrates the percentage of fixations that were directed to the new object, broken down by condition. On average, $45 \%$ of the first four fixations following the scene change were directed to the new object if it appeared during a 
Probability of First Look to New Object
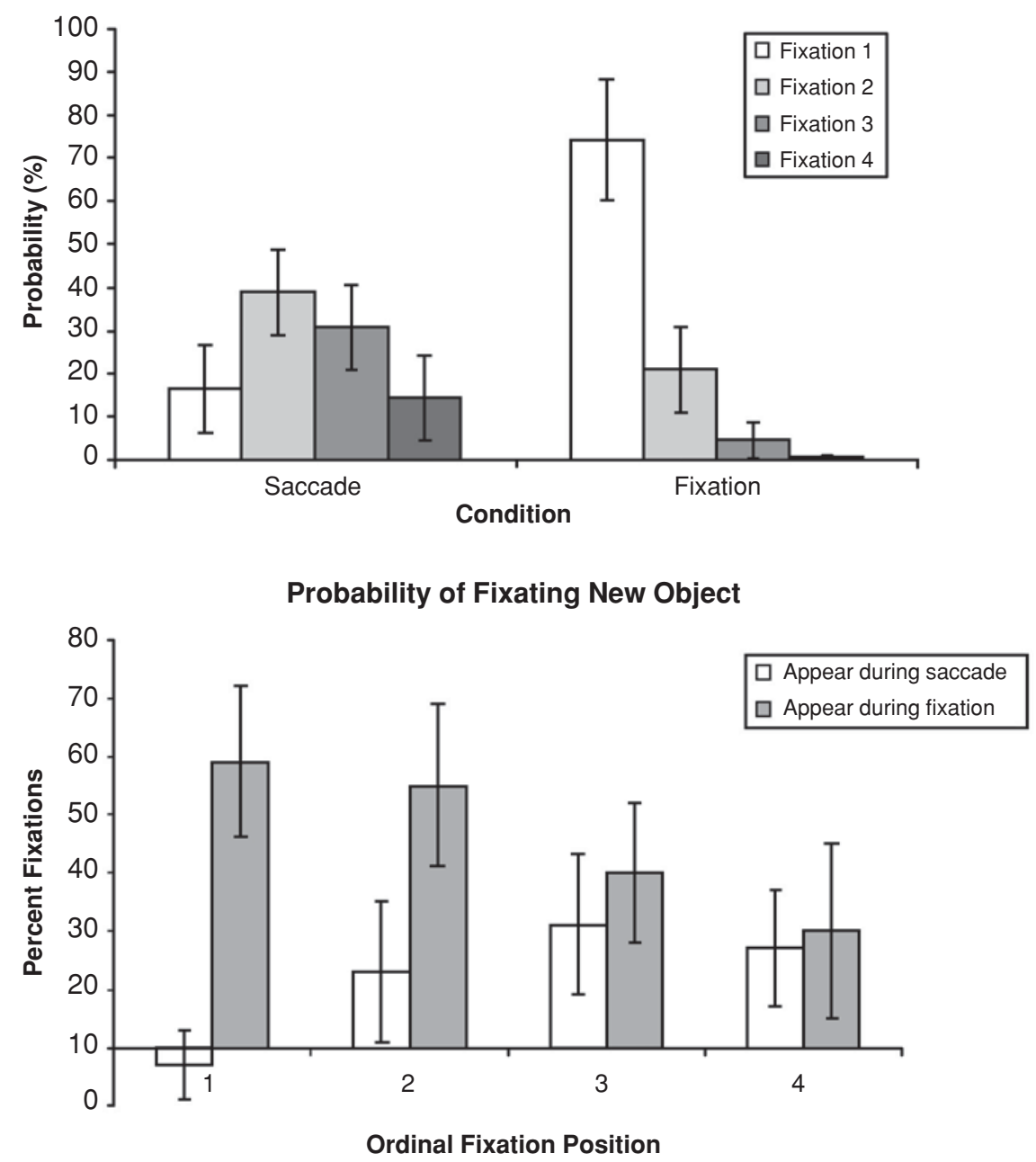

Figure 2. (Top) The probability that the first look to the new object occurred at each of the first four fixations after its appearance. (Bottom) The mean probability that fixations just after the new object appeared were localized on the new object. The $x$-axis crosses the $y$-axis at the baseline rate of viewing (chance). All error bars represent $95 \%$ confidence intervals.

fixation. In contrast, $22 \%$ of these fixations targeted the new object if it appeared during a saccade. This difference was reliable $\left[F(1,7)=15.8, M S_{\mathrm{e}}=570.8, p<.01\right]$. For comparison, the chance probability of viewing the critical object was established by observing the percentage of fixations directed to the object in the offset condition prior to its removal from the scene. This baseline rate was $10 \%$. Ninety-five percent confidence intervals indicated that for both the saccade and the fixation conditions, the new object was fixated more frequently at all ordinal fixation positions, relative to baseline, except for Fixation 1 in the saccade condition, suggesting that the appearance of the new object captured attention immediately in the fixation condition, but not in the saccade condition (Brockmole \& Henderson, 2005).

A main effect of ordinal fixation position indicated that the new object was not fixated equally at all ordinal fixation positions $\left[F(3,21)=3.13, M S_{\mathrm{e}}=92.5, p<.05\right]$, and a reliable interaction between onset condition and ordinal fixation position indicated that viewing patterns differed between the fixation and the saccade conditions $\left[F(3,21)=16.5, M S_{\mathrm{e}}=118.4, p<.001\right]$. The probability of fixating the new object when it occurred during a fixation decreased from 59\% at Fixation 1 to $30 \%$ at Fixation 4. The opposite effect was observed in the saccade condition, where the probability of fixating the new object increased from $7 \%$ at Fixation 1 to $27 \%$ at Fixation 4. These results also suggest that new objects immediately capture attention in the fixation condition, but not in the saccade condition.

In summary, suddenly appearing new objects were fixated more often than would be expected by chance upon their appearance. Consistent with the speed with which new objects were detected in the fixation and saccade conditions, above-baseline viewing rates emerged later in the saccade condition. In addition, a new object accompanied 
by a transient signal drew the eyes approximately twice as often, as compared with a nontransient new object.

\section{Disappearing Objects}

Number of eye movements to first fixation on location of deleted object. On average, the location of the deleted object was fixated 3.04 saccades after the object's disappearance. Deletions that occurred during a fixation ( $M=1.94$ saccades after disappearance; cf. 1.57 saccades after appearance) were viewed sooner than those that occurred during a saccade $[M=4.14$ saccades after disappearance; cf. 3.01 saccades after appearance; $t(7)=$ $3.62, p<.01]$. No effects in a between-subjects analysis were reliable, (all $p \mathrm{~s}>.15$ ), indicating no differences in the overall speed with which object deletions and additions were detected.

The probability that the first look to the removed object occurred at each of the first four ordinal fixation positions differed $\left[F(3,21)=10.5, M S_{\mathrm{e}}=1.21, p<.001\right]$, and these differences were not equal in the saccade and the fixation conditions $\left[F(3,21)=11.5, M S_{\mathrm{e}}=1.56, p<\right.$
.001 ; see Figure $3 \mathrm{~A}]$. In the fixation condition, $57 \%$ of first looks to the region of deletion occurred at Fixation 1, followed by a rapid decline in the probability of a first look at each of the next ordinal fixation positions. Only $14 \%$ of first looks to the deletion occurred at Fixations 3 and 4 combined. Comparatively, detection of object deletions that occurred during a saccade was extended in time, with most first looks occurring at Fixations +2 and +3 , each accounting for $31 \%$ of first looks to the location of the removed object. Detection of the disappearing objects was extended in time if it happened during a saccade.

Probability of fixating location of deleted object. On average, $25 \%$ of the first four fixations following the disappearance of an object were directed to the location of the deletion if it occurred during a fixation. In contrast, $12 \%$ of fixations were directed to the deletion if it occurred during a saccade. This difference was reliable $\left[F(1,7)=18.7, M S_{\mathrm{e}}=132.9, p<.01 ;\right.$ see Figure 3B $]$. In addition, a between-subjects comparison showed that these rates of fixating the region of deletion were reliably lower than those of fixating an addition to the scene
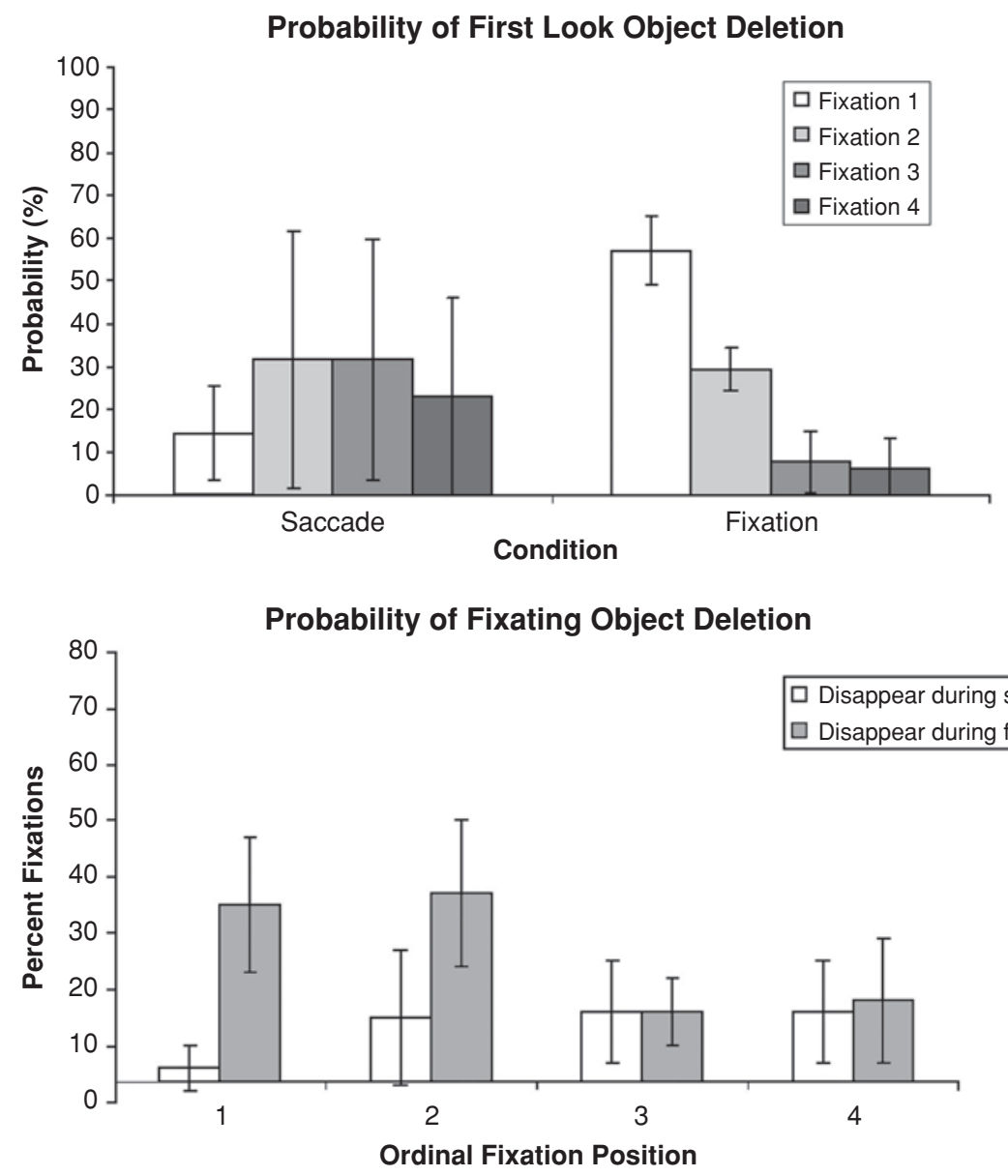

Figure 3. (Top) The probability that the first look to the region of object deletion occurred at each of the first four fixations after the critical object's disappearance. (Bottom) The mean probability that fixations just after the offset were localized on the region once containing the removed object. The $x$-axis crosses the $y$-axis at the baseline rate of viewing (chance). All error bars represent $95 \%$ confidence intervals. 
$\left[F(1,14)=16.7, M S_{\mathrm{e}}=411.3, p<.01 ;\right.$ no betweensubjects interactions were reliable]. However, the chance probability of viewing the location of the critical object when it was absent from the scene, established by observing the percentage of fixations directed to this area in the new-object condition prior to the new object's appearing, was $4 \%$. Ninety-five percent confidence intervals indicated that the probability of fixating the location of the deletion was above this baseline rate at all ordinal fixation positions in the fixation condition and at Fixation Positions +3 and +4 in the saccade condition. Although object disappearances were detected less frequently than appearances, they were still fixated at rates that were at least three times baseline.

Regions of deletion were not fixated equally at all ordinal fixation positions $\left[F(3,21)=2.80, M S_{\mathrm{e}}=88.1\right.$, $p<.06]$, and critically, the effect of fixation position was not equal across the fixation and the saccade conditions $\left[F(3,21)=7.4, M S_{\mathrm{e}}=122.2, p<.01\right]$. The probability of fixating the region of deletion when it occurred during a fixation decreased from $35 \%$ at Fixation 1 to $18 \%$ at Fixation 4. The opposite effect was observed in the saccade condition, in which the probability of fixating the deletion increased from $6 \%$ at Fixation 1 to $16 \%$ at Fixation 4. In summary, regions once housing objects that suddenly disappeared were fixated more often than would be expected by chance upon their disappearance, but less often than when the object suddenly appeared in the scene. In addition, above-baseline viewing rates emerged later in the saccade condition, and a deletion accompanied by a transient signal drew the eyes approximately twice as often, as compared with a nontransient disappearance of an object.

Appearing versus disappearing objects. Both the appearance and the disappearance of an object embedded in a real-world scene were prioritized over other objects, although prioritization in both cases was faster and more frequent if the scene change occurred during a fixation, as compared with during a saccade. These results suggest that both the addition and the removal of an object from a scene are powerful cues that the attention system uses to guide gaze. Regardless of whether the scene change occurred during a fixation or a saccade, however, additions were attended twice as often as deletions, raising the question of why new objects enjoy a greater degree of influence on attention. One possibility is that observers prefer to fixate objects, rather than backgrounds (Henderson \& Hollingworth, 1998). Given this bias, the appearance of an object may be more cognitively salient than the removal of an object: Object additions always produce an informative object for observers to fixate, whereas the removal of an object will often reveal noninformative background regions of a scene. As a result, new objects would attract the eyes more often than would object deletions.

To test this object versus background hypothesis, we took a closer look at the object deletion condition in this experiment. Of the 30 stimuli used in this experiment, 15 of the critical objects occluded background elements, and 15 occluded parts of other objects, so that they were partly visible and, therefore, known to exist (only 1 of these stimuli occluded an entire object). We therefore divorced situations in which an object was removed from the scene and those in which objects were removed and added simultaneously. If prioritization is driven by the type of information revealed by a scene change (i.e., objects or backgrounds), greater prioritization should be observed in cases in which once occluded objects are fully revealed. Although objects are preferred over backgrounds when static displays are viewed, if objects revealed through the removal of an occluder are fixated more often than in cases in which the occluder was never present, some information about the dynamic change must have guided fixation to the critical region over and above what would be expected in a static nonchanging display.

Indeed, more prioritization was observed if objects were revealed than if backgrounds were revealed $[F(1,7)=$ $\left.30.8, M S_{\mathrm{e}}=91.1, p<.01\right]$. Interactions between occlusion type (object vs. background) and scene change condition $\left[F(1,7)=4.43, M S_{\mathrm{e}}=150.3, p<.07\right]$, as well as ordinal fixation position $\left[F(3,21)=8.45, M S_{\mathrm{e}}=\right.$ $3.80, p<.03$ ], were observed (see Table 1). In the fixation condition, the region of deletion was fixated at rates greater than chance, regardless of whether the removal of the critical object revealed objects or backgrounds, but the revelation of once occluded objects drew the eyes approximately twice as often. Note, however, that the extent of prioritization given the disocclusion of an object $(32 \%$ of fixations in the critical region, averaged across ordinal

Table 1

Mean Probabilities (With Standard Errors) of Fixating the Region of an Object Disappearance Broken Down by Occlusion Type, Onset Condition, and Ordinal Fixation Position

\begin{tabular}{|c|c|c|c|c|c|c|c|c|}
\hline \multirow[b]{3}{*}{ Conditions } & \multicolumn{8}{|c|}{ Ordinal Fixation Position } \\
\hline & \multicolumn{2}{|c|}{+1} & \multicolumn{2}{|c|}{+2} & \multicolumn{2}{|c|}{+3} & \multicolumn{2}{|c|}{+4} \\
\hline & $M$ & $S E$ & $M$ & $S E$ & $M$ & $S E$ & $M$ & $S E$ \\
\hline \multicolumn{9}{|c|}{ Occluded background } \\
\hline Fixation & 20.0 & $5.62^{*}$ & 24.2 & $4.83^{*}$ & 13.5 & $3.83^{*}$ & 16.1 & $5.08^{*}$ \\
\hline Saccade & 5.43 & 2.68 & 8.33 & 4.17 & 16.6 & $4.37^{*}$ & 9.4 & 4.84 \\
\hline \multicolumn{9}{|l|}{ Occluded objects } \\
\hline Fixation & 48.6 & $4.42^{*}$ & 49.4 & $7.09^{*}$ & 15.6 & $3.82^{*}$ & 15.9 & $5.43^{*}$ \\
\hline Saccade & 3.35 & 2.20 & 18.0 & $6.30^{*}$ & 15.1 & $4.74^{*}$ & 22.5 & $4.23^{*}$ \\
\hline
\end{tabular}

*Greater than baseline, $p<.05$. 
fixation positions) was less than that observed in the newobject condition ( $46 \%$ of fixations in critical region). This pattern may be attributed to the fact that additions always constituted an entirely new object that could not be anticipated, whereas deletions revealed only parts of objects that were known to exist.

In the saccade condition, the region of deletion was also fixated if the removal of the critical object disoccluded other objects, but generally, deletions that revealed background elements were not prioritized (Fixation +3 constitutes an exception). Without a transient signal, object deletions affected gaze more reliably if their disappearance revealed detail about objects that was not visible prior to the deletion. In this condition, then, object deletions were very weak attractors of attention when they were not accompanied by a corresponding appearance of once occluded objects. Given Brockmole and Henderson's (2005) conclusion that this condition reflects a memory-guided prioritization process, the percept-to-memory comparison involved in the detection of nontransient scene changes does not involve notice of the disappearance of objects unless new object features are revealed in the process.

We propose that the limitations of visual short-term memory (VSTM) may be involved in this distinction. As scene viewing progresses, VSTM must be updated with new information. Given a limited capacity, the updating processes may maintain existing information in the VSTM that remains momentarily relevant, transfer information to long-term memory, or allow older, less relevant information to decay. Results in the memory literature suggest that participants are less likely to detect deletions than to detect additions, perhaps because there is no retrieval cue in the deletion condition (see, e.g., Hearst, 1991; Henderson et al., 2003; Pezdek et al., 1988), causing a failure to retrieve information from short- or long-term memory. This explanation would also account for the better detection of an object deletion that reveals an occluded object than of one that reveals backgrounds. Recent work in spatial processing is also consistent with this view. During navigation, observers keep track of objects or places they are approaching (those that they can see) and lose track of objects or places that they have passed (those that they cannot see; Wang \& Brockmole, 2003). Extrapolating this result to more conventional tests of visual short-term memory opens new and interesting questions regarding what information is selected for encoding in memory and how that information is updated as the contents of a scene change.

\section{REFERENCES}

Bacon, W. F., \& Egeth, H. E. (1994). Overriding stimulus-driven attentional capture. Perception \& Psychophysics, 55, 485-496.

Brockmole, J. R., \& Henderson, J. M. (2005). Prioritization of new objects in real-world scenes: Evidence from eye movements. Journal of Experimental Psychology: Human Perception \& Performance, 31, 857-868.

Chastain, G., \& Cheal, M. (1999). Time course of attention effects with abrupt-onset and offset single- and multiple-element precues. American Journal of Psychology, 112, 411-436.
Folk, C. L., Remington, R. W., \& Johnston, J. C. (1992). Involuntary covert orienting is contingent on attention control settings. Journal of Experimental Psychology: Human Perception \& Performance, 18, 1030-1044

Franconeri, S. L., \& Simons, D. J. (2003). Moving and looming stimuli capture attention. Perception \& Psychophysics, 65, 999-1010.

Hearst, E. (1991). Psychology and nothing. American Scientist, 79, 432-443.

Henderson, J. M., \& Hollingworth, A. (1998). Eye movements during scene viewing: An overview. In G. Underwood (Ed.), Eye guidance in reading and scene perception (pp. 269-293). Amsterdam: Elsevier.

Henderson, J. M., Williams, C. C., Castelhano, M. S., \& Falk, R. J. (2003). Eye movements and picture processing during recognition. Perception \& Psychophysics, 65, 725-734.

JoNIDES, J., \& YANTIS, S. (1988). Uniqueness of abrupt visual onset in capturing attention. Perception \& Psychophysics, 43, 346-354.

MiLlER, J. (1989). The control of attention by abrupt visual onsets and offsets. Perception \& Psychophysics, 45, 567-571.

Pezdek, K., Maki, R., Valencia-Laver, D., Whetstone, T., Stoeckert, J., \& Dougherty, T. (1988). Picture memory: Recognizing added and deleted details. Journal of Experimental Psychology: Learning, Memory, \& Cognition, 14, 468-476.

Pratt, J., \& Hirshhorn, M. (2003). Examining the time course of facilitation and inhibition with simultaneous onset and offset cues. Psychological Research, 67, 261-265.

TheEuwes, J. (1991). Exogenous and endogenous control of attention: The effect of visual onsets and offsets. Perception \& Psychophysics, 49, 83-90.

THEEUwES, J. (1994). Stimulus-driven capture and attentional set: Selective search for color and visual abrupt onsets. Journal of Experimental Psychology: Human Perception \& Performance, 20, 799-806.

Theeuwes, J., Kramer, A. F., Hahn, S., \& Irwin, D. E. (1998). Our eyes do not always go where we want them to go: Capture of the eyes by new objects. Psychological Science, 9, 379-385.

WANG, R. F., \& BRockmole, J. R. (2003). Human navigation in nested environments. Journal of Experimental Psychology: Learning, Memory, \& Cognition, 29, 398-404.

Watson, D. G., \& Humphreys, G. W. (1995). Attention capture by contour onsets and offsets: No special role for onsets. Perception \& Psychophysics, 57, 583-597.

YAnTIS, S., \& Jonides, J. (1984). Abrupt visual onsets and selective attention: Evidence from selective search. Journal of Experimental Psychology: Human Perception \& Performance, 22, 1505-1513.

\section{NOTES}

1. On average, luminance within the critical regions was unchanged by the appearance or disappearance of an object. Thus, changes in luminance could not contribute to capture effects in this study.

2. In a previous study (Brockmole \& Henderson, 2005) in which appearing objects were used, observers searched for and identified the new objects. Although the observers could often verbally identify the object, we found no differences in their gaze patterns, as compared with cases in which the observers were instructed to memorize the scene.

3. On average, the onset/offset occurred $5.98 \mathrm{sec}$, or 16.8 saccades, into viewing. These values did not vary significantly across experimental factors.

4. The change was successfully accomplished during a fixation on $94 \%$ of the fixation trials and during a saccade on $83 \%$ of the saccade trials. These rates did not vary significantly across experimental factors. The remaining trials were excluded from the reported analyses.

5. To avoid issues of multicolinearity introduced by expressing the number of first looks to the new object at each ordinal fixation position as a conditional probability, the ANOVAs were performed on the raw number of times the first look occurred at each fixation position.

(Manuscript received September 7, 2004; revision accepted for publication March 2, 2005.) 